\title{
Case Report: Diagnosis of COVID-19 versus Tropical Diseases in Pakistan
}

\author{
Syed Muhammad Mashhood Ali Bokhari, ${ }^{1 *}$ Fatima Mahmood, ${ }^{1}$ and Syed Muhammad Saud Ali Bokhari ${ }^{2}$ \\ ${ }^{1}$ Nishtar Hospital, Multan, Pakistan; ${ }^{2}$ Shifa College of Medicine, Islamabad, Pakistan
}

\begin{abstract}
A25-year-old medical student presented in Multan, Pakistan with a high fever, cough, myalgia, and diarrhea consistent with the typical signs and symptoms of novel coronavirus disease (COVID-19). The patient had traveled to high COVID-19-risk areas within Pakistan and had no significant medical and surgical history. Based on nasopharyngeal and oropharyngeal swab testing, the patient was found to be negative for COVID-19. He subsequently developed a diffuse rash and had serology consistent with dengue and measles. The patient was treated symptomatically, and his condition gradually improved over 7 days. This case highlights the high prevalence of many tropical diseases in low-income countries and the need for clinicians to consider alternate diagnoses in addition to testing for COVID-19 during the pandemic.
\end{abstract}

\section{CASE}

A 25-year-old man presented to the emergency department of Nishtar Hospital, Multan, Pakistan with a 3-day history of high fever, chills, dry cough, myalgias, and diarrhea. He reported a sudden onset of symptoms. The patient is a medical student with no prior medical history. He reported recent travel with his family to Islamabad and Karachi, regions with known novel coronavirus disease (COVID-19) transmission, but family members denied similar complaints. The patient's childhood and adult immunization records showed that all immunizations were up to date.

Considering the emergence of COVID-19 and the patient's travel history, he was admitted to the hospital. Droplet and contact precautions were initiated. The staff caring for the patient were provided appropriate personal protective equipment.

In the ward, the patient was continuously shivering, with a temperature of $39.5^{\circ} \mathrm{C}$, pulse 140 per minute, blood pressure $95 / 60 \mathrm{~mm} \mathrm{Hg}$, respiratory rate 18 per minute, and oxygen saturation $99 \%$ while breathing ambient air. Breath sounds were equal and clear in both lung fields. Chest radiographs showed no abnormalities.

Initial laboratory tests were mostly unremarkable but showed mild elevation of liver function tests (Table 1). Nasopharyngeal and oropharyngeal swabs were tested for severe acute respiratory syndrome-coronavirus-2 by reverse transcriptasePolymerase chain reaction, and both tests were negative. Therefore, we decided to steer the patient's management toward other infectious diseases within our differential diagnosis.

The patient was treated with intravenous hydration, ceftriaxone, metronidazole, and paracetamol. Initially, he experienced relapsing high fevers, productive cough, severe myalgias, and worsening diarrhea. On the third day of hospital admission, the patient developed a maculopapular rash on the face and trunk, which gradually spread to the extremities over 2 days. To evaluate the rash, measles and varicella antibody tests were also conducted.

Microscopy of thick and thin smears for malaria, serology and surface antigen markers for viral hepatitis, and bacterial blood and stool cultures were negative. Testing for dengue

*Address correspondence to Syed Muhammad Mashhood Ali Bokhari, Nishtar Hospital, Nishtar Rd., Multan 60000, Pakistan. E-mail: mashhood.bokhari@hotmail.com showed positive IgM titers (1.6 U/L) and borderline NS1 antigen $(0.8 \mu \mathrm{g} / \mathrm{mL})$ results. Further serologic investigation revealed elevated IgM (137 U/L) against measles virus and negative serology for varicella. The same treatment measures were continued. By day 7 , the patient's fever, rash, and diarrhea had subsided; intravenous access was removed; and he was discharged.

\section{DISCUSSION}

The WHO defines tropical diseases as infectious diseases that thrive in hot and humid tropical conditions, including dengue, malaria, leishmaniasis, schistosomiasis, and onchocerciasis, among several others. ${ }^{1}$ Despite medical advancements and numerous control measures, many developing nations still face the burden of tropical diseases. Dengue fever is one of the most prevalent diseases in Pakistan, with more than 50,000 cases and resulting in more

TABLE 1

Clinical laboratory results

\begin{tabular}{lccc}
\hline \multicolumn{1}{c}{ Variable } & Reference range & Hospital day 1 & Hospital day 3 \\
\hline Sodium $(\mathrm{mmol} / \mathrm{L})$ & $136-145$ & 137 & 138 \\
Potassium $(\mathrm{mmol} / \mathrm{L})$ & $3.5-5.1$ & 3.2 & 3.3 \\
Chloride $(\mathrm{mmol} / \mathrm{L})$ & $98-107$ & 101 & 100 \\
Creatinine $(\mathrm{mg} / \mathrm{dL})$ & $0.7-1.3$ & 0.73 & - \\
ESR $(\mathrm{mm} / \mathrm{hour})$ & $1-20$ & 28 & - \\
Total protein $(\mathrm{g} / \mathrm{dL})$ & $6.0-8.5$ & 6.1 & - \\
Albumin $(\mathrm{g} / \mathrm{dL})$ & $3.2-4.8$ & 3.3 & - \\
Aspartate aminotransferase & $<35$ & 83 & - \\
$\quad(\mathrm{U} / \mathrm{L})$ & & & \\
Alanine aminotransferase & $<45$ & 95 & - \\
$\quad(\mathrm{U} / \mathrm{L})$ & & & \\
Alkaline phosphatase $(\mathrm{U} / \mathrm{L})$ & $50-116$ & 112 & - \\
Lactate dehydrogenase & $120-246$ & 240 & - \\
$\quad(\mathrm{U} / \mathrm{L})$ & & & \\
Total bilirubin (mg/dL) & $0.2-1.1$ & 0.2 & - \\
Hb $(\mathrm{g} / \mathrm{dL})$ & $13-18$ & 14.4 & 14.2 \\
Hct $(\%)$ & $38-52$ & 42 & 44 \\
Platelets $\left(\times 10^{9} / \mathrm{L}\right)$ & $150-400$ & 157 & 145 \\
White blood cell count & $4-11$ & 7.4 & 8 \\
$\quad\left(\times 10^{9} / \mathrm{L}\right)$ & & & \\
Neutrophils $(\%)$ & $40-75$ & 50 & 52 \\
Lymphocytes $(\%)$ & $20-50$ & 35 & 34 \\
Monocytes $(\%)$ & $2-10$ & 10 & 8 \\
Eosinophils $(\%)$ & $1-6$ & 5 & 6 \\
INR & $0.8-1.1$ & 1.0 & - \\
\hline ESR = erythrocyte sedimentation rate; & INR $=$ international normalized ratio. &
\end{tabular}


TABLE 2

Microbiology report

\begin{tabular}{lcl}
\hline \multicolumn{1}{c}{ Test } & Reference range & \multicolumn{1}{c}{ Results } \\
\hline Novel coronavirus disease & - & Negative \\
$\quad$ RT-PCR test & \multicolumn{2}{l}{1.59} \\
Dengue virus IgM $(\mathrm{U} / \mathrm{L})$ & $<0.9$ & \multicolumn{1}{c}{0.8} \\
Dengue NS1 antigen $(\mu \mathrm{g} / \mathrm{mL})$ & $<0.6$ & \multicolumn{1}{c}{136.6} \\
Measles IgM $(\mathrm{U} / \mathrm{mL})$ & $<20$ & Negative \\
Influenza & - & No growth after 7 days \\
Stool culture & - & No growth after 7 days \\
Blood culture & - & Negative \\
Thick and thin smear & - & \\
$\quad$ microscopy for malaria & &
\end{tabular}

than 90 deaths in $2019 .^{2}$ Multan district faced a devastating outbreak of dengue fever in 2015 . $^{3}$

In our case, the patient raised suspicion for concomitant measles infection based on the WHO clinical case definition, which defines suspected measles as fever and maculopapular rash with cough, coryza, or conjunctivitis. ${ }^{4}$ Although the appearance of high titers of measles IgM seems puzzling in an immunocompetent, immunized patient, possible explanations for infection could be waning immunity, secondary vaccine failure, or prolonged exposure to a measles patient. ${ }^{5}$ Aggressive vaccination campaigns against measles were accompanied by a $71 \%$ reduction in mortality and morbidity rates worldwide between 2000 and 2011, but Pakistan continues to face this disease burden, with 94 measles deaths in Punjab during early $2013 .^{6}$

Although COVID-19 is emerging as a pressing threat worldwide, this case underscores the need for clinicians practicing in Pakistan and other tropical regions to consider the possibility of common tropical diseases in all suspected patients. It also highlights the challenges facing the measles elimination campaign and the common threat of dengue in Pakistan.

Received April 25, 2020. Accepted for publication April 30, 2020.

Published online May 5, 2020.

Acknowledgments: Publication charges for this article were waived due to the ongoing pandemic of COVID-19.

Authors' addresses: Syed Muhammad Mashhood Ali Bokhari and Fatima Mahmood, Nishtar Hospital, Multan, Pakistan, E-mails: mashhood.bokhari@hotmail.com and fatimamahmood027@gmail. com. Syed Muhammad Saud Ali Bokhari, Shifa College of Medicine, Islamabad, Pakistan, E-mail: m.saudbukhari@gmail.com.

This is an open-access article distributed under the terms of the Creative Commons Attribution (CC-BY) License, which permits unrestricted use, distribution, and reproduction in any medium, provided the original author and source are credited.

\section{REFERENCES}

1. Ross R, 1901. Tropical diseases. J Am Med Assoc XXXVII: 450-451.

2. World Health Organization, 2019. Epidemic and pandemic-prone diseases Outbreak update - Dengue in Pakistan, 1 December 2019 [Internet]. Available at: http://www.emro.who.int/pandemicepidemic-diseases/dengue/outbreak-update-dengue-in-pakistan1-december-2019.html.

3. Saba S, Khan AUR, Naeem-Ullah U, Bokhari SHM, 2019. Clinical profiles of dengue fever patients, during an outbreak. J Arthropod Borne Dis 13: 126-134.

4. Dietz FJ, Nieburg P, Gubler DJ, Gomez I, 1992. Diagnosis of measles by clinical case definition in dengue-endemic areas: implications for measles surveillance and control. Bull World Health Organ 70: 745-750.

5. Hickman CJ et al., 2011 Laboratory characterization of measles virus infection in previously vaccinated and unvaccinated individuals. J Infect Dis 204 (Supp/ 1): 549-558.

6. Khan T, Qazi J, 2014. Measles outbreaks in Pakistan: causes of the tragedy and future implications. Epidemiol Rep 2: 1. 